\title{
THE MECHANISM THROUGH WHICH LIGHT AND HEAT INFLUENCE GENETIC FACTORS FOR WING DEVELOPMENT IN APHIDS ${ }^{1,2}$
}

\author{
A. FRANKLIN SHULL \\ Department of Zoology, University of Michigan, Ann Arbor
}

In the extensive work of physiological genetics and experimental morphogenesis, it has seldom been possible to produce precisely the same sharply defined structural modification by two different environmental agents. That favorable situation exists in certain strains of aphids, in which wing production can be completely stopped by either high temperature or continuous light.

It has been uncertain whether these two agents work through the same physiological mechanism, or through different mechanisms. If through different ones, proof of that fact would have an important bearing upon the reality of a genuine single time of determination of wings, especially if the times at which the two agents work could be rather accurately ascertained. Producing their effects at the same stage would favor the view that there is a real time of determination of wing's perhaps wholly independent of the nature of any agent which could interfere with their production. If light and temperature operate through the same mechanism, establishment of that fact would be a first step in the discovery of the nature of that mechanism. It would have to be of a sort which might respond to both of these physical agents.

The chief means of distinguishing between the two possibilities just indicated is to ascertain the stage at which they

' Contribution from the Department of Zoology, University of Michigan.

2 The computations involved in this investigation were made with a calculating machine provided by the trustees of the Joseph Henry Fund. Aid was also received from the Faculty Research Fund of the University of Michigau. 
are effective, and the speed with which they act. How long must the agents be applied before they influence wing production, and before what stage must this application be completed in order to be effective with respect to a given individual? It is to be expected that in part these questions may prove to be inseparable.

\section{METHOD OF ASCERTAINING SPEED OF ACTION}

The influence of both light and temperature on wing production in the aphid Macrosiphum solanifolii is exerted only before birth; attempts to produce the same results later have always failed. It is necessary, therefore, to apply the agents to the parents. In the experiments the parents were changed from intermittent light ( 8 hours of light alternating repeatedly with 16 hours of darkness) to continuous light, or firom a moderate temperature $\left(20^{\circ} \mathrm{C}\right.$.) to a high temperature (usually $30^{\circ}$ ). The offspring born after the change were collected in successive lots by transferring the parents from plant to plant; offspring were thus allowed to grow to maturity on the plant on which they were born, while the parents were always on fresh young plants.

The length of time during which the parents remained on one plant varied in the different experiments, and often at different times in the same experiment. Usually the period on the first plant after the change of light or temperature was 12 hours. If the suppression of wings was expected to be rapid (as with high temperature), the succeeding periods were likewise about 12 hours. If the stoppage of wing production was likely to be slow, the period on each plant after the first was often longer -24 hours or even more. After such longer periods, the final periods were sometimes shortened again, to sharpen the definition of the time required to complete the process of suppression.

The offspring produced on each of the successive plants were reared to maturity, and the number of winged and wingless aphids in each lot recorded. A number of different ways of computing the length of time required to suppress wings 
were considered. No experiment was used unless the intermittent light applied prior to its beginning had resulted in the development of wings in all individuals. Either the last plant before the change of light or temperature, or the first plant after that change, must bear only winged offspring, or that experiment was rejected as inconclusive. Under these circumstances, the time at which the first wingless aphid was born after the change might have been taken as the measure of the speed of the suppression of wings. This method was rejected because it was never known precisely when in the 12- or 24-hour period it was produced, and any erroneous assumption made concerning it could not be balanced statistically by corresponding assumptions regarding many other individuals. As one alternative, the time at which wing production completely ceased could have been taken as the measure of the speed of suppression of wings. This plan was rejected because it gave no weight to the distribution of the numbers of winged and wingless between the beginning and end of the process, and this distribution was found to vary considerably.

Some measure of central tendency is required. It is probably essential that in computing this measure the components be not weighted with the number of offspring produced; for, while the extent of suppression of wings at any moment would be more accurately computed from many aphids than from few, it seems unlikely that the suppression is in any way connected with rate of reproduction. It is the extent to which the power to suppress wings has progressed at any given time, not the number of offspring, which is important.

Accordingly, the numbers of wingless aphids in the successive lots of offspring were reduced to percentages. Each percentage was associated with a given number of hours after the change of light or temperature. There had been no suppression at all up to the end of the period during which the last exclusively winged progeny was produced. In each of the following periods in which there was a mixed progeny, the entire progeny was considered to be concentrated at the middle 
point of the period. Finally, suppression of wings was complete at the beginning of the first period in which an exclusively wingless progeny was produced.

The pairs of times and percentages could be represented by points related to a pair of coordinate axes. In every experiment these points would indicate, irregularly, a rising trend of winglessness. This trend was ascertained by computing the equation of the straight line best representing all the points, the criterion of "best" being the least sum of the squares of their deviations from the line. By means of this equation, which was in the form for predicting hours from percentages, there was computed the number of hours after the change of light or temperature at which $50 \%$ of the offspring should be wingless.

This method of determining a "mean" has the advantage that it can be used in experiments which were, through mistaken judgment, discontinued before wings were wholly suppressed. A trend established by the first half dozen or more times and percentages would presumably be little changed by the addition of one, or sometimes two, additional pairs of values. The data from the investigation as a whole were thus appreciably enlarged.

\section{SPEED OF ACTION OF IIIGI TEMPERATURE}

One of the stocks of aphids was regularly kept at about $20^{\circ} \mathrm{C}$. and continuous (electric) light. In preparation for a temperature experiment, a group of young aphids was placed, beginning usually a day or two after birth, in darkness 16 hours out of each 24. By the time they were adult they had usually been in this intermittent light long enough that all their early offspring were winged. They were then changed to $30^{\circ}$, but the alternation of light and darkness was continued. In the experiments now being described the temperature was raised at the moment when the aphids were changed from darkness to light. The length of time required to effect a $50 \%$ suppression of wings was then determined as described in the preceding section. 
This experiment was performed seventeen times. The mean period elapsing from the change of temperature to $50 \%$ wing suppression was $24.07 \pm 0.8$ hours.

\section{SPEED OF ACTION OF CONTINUOUS LIGHT}

Starting with young aphids of the same $20^{\circ}$ stock described in the preceding section, a group was placed in alternating light and darkness, just as for a temperature experiment. After they had reproduced, to prove the efficacy of the intermittent light in permitting wing development, they were changed to continuous light. The time elapsing from the last removal from darkness into light, to the time at which $50 \%$ of wing production had been suppressed, was determined. This experiment was performed forty-one times, and the mean period required for the one-half reduction of wings was $71.4 \pm 1.8$ hours. This was longer than had been expected on the basis of less exact tests with another strain of aphids some years earlier. How much of this increase in the length of time required to suppress wings, as compared with earlier experiments, is a result of the long preceding period in alternating light and darkness (see later in this paper), it is now impossible to say. In the present experiments, beginning the intermittent-light treatment a day or two after birth meant that it continued about 8 days. In the old experiments that treatment could seldom have been so long. It is possible, of course, that the strain of aphids now being used responds less quickly; considerable differences between strains in the speed of this reaction are definitely known to exist.

IMMEDIATE ACTION OR PERIOD OF PREPARATION?

The results given in the two preceding sections, indicating that $30^{\circ}$ temperature suppresses wings in about one third of the time required by continuous light, are open to two possible interpretations. The environmental agent either acts directly on the thickening of the hypodermis which is the start of wing development, or builds up some physiological condition (per- 
haps accumulates some chemical substance) which is in turn the agent working directly on wings.

If the action of light and temperature is direct, it would presumably be immediate. 'The body of an aphid is rather transparent, and so small that it must acquire promptly the temperature of the surrounding air and objects. Under these circumstances the difference in the length of time required for light and temperature to stop wing production should mean (with direct action) that these two agents interfere with wing production or determination at two different times. Light should be critical roughly 2 days $(71.4-24.7=46.7$ hours $)$ earlier than high temperature in this particular strain of aphids.

If the action of light and temperature is indirect, if they must build up a physiological state or cause the production of some inhibiting substance, the difference in the time of final result could relate entirely to the time required to effect this preparation. The time of determination of wings, the critical moment of interference with their development, might then be the same for both agents.

Some information believed to bear on these two alternative hypotheses is derived from tests of different high temperatures. Experiments were conducted in the same way as described in the section on high temperature, except that the aphids were removed from $20^{\circ}$ to $29^{\circ}$, to $28^{\circ}$ or to $26^{\circ}$. The $29^{\circ}$ temperature was used in thirteen experiments, $28^{\circ}$ in nine experiments, $26^{\circ}$ in six. The time required to attain a $50 \%$ reduction of wing production was determined in the usual manner, with the following results:

\footnotetext{
$30^{\circ}$ reduced wing production one half in $24.7 \pm 0.8$ hours

$29^{\circ}$ reduced wing production one half in $32.9 \pm 1.3$ hours

$28^{\circ}$ reduced wing production one half in $42.3 \pm 1.5$ hours

$26^{\circ}$ reduced wing production one half in $77.4 \pm 2.6$ hours
}

Each of the three highest temperatures suppressed wings completely in any experiment which was continued as long as \pm days. Some of the $26^{\circ}$ experiments completed the sup- 
pression in a somewhat longer time, others did not complete it at all before the end of the experiment, and there is no assurance that they would have done so even if the offspring had been preserved up to the time of death of the parents. This is in close agreement with earlier experiments ${ }^{3}$ with continuous high temperature in which it was shown, in another strain, that temperatures of $26^{\circ}$ and above suppressed wings entirely. The earlier experiments did not test the speed with which that result was attained.

Different intensities of light have not been tested with the present strain of aphids, but in the earlier experiments referred to above intensities ranging from 10,000 meter-candles down to 5 meter-candles were used. The lowest of these intensities, used intermittently, did not cause $100 \%$ wing production. The other intensities, from 37 meter-candles up, used intermittently, all did result in exclusively winged progeny, but the lower the intensity the longer the time required.

Any agent which acts directly on an embryonic change, not through a preparatory process, should affect that development at once. The fact that three high temperatures, though completely effective in the end, require different times to become effective, indicates that something must first be built up. Five different intensities of intermittent light, in the earlier experiments, caused complete wing production eventually, but they required different lengths of time to do so. Again there must be a preparatory process which the light requires time to effect.

Another test of the existence of a preparatory process should also be found in the extent of the effects produced in different lengths of time. If continuous light causes the accumulation of a substance which suppresses wings, and alternating light and darkness dissipate or destroy that substance, it should be possible to carry these changes far beyond the limits that can be detected by experiment. The inhibiting substance conld presumably exist in a concentration much higher

${ }^{3}$ Shull, A. F. 1929. Roux's Arch. f. Entwick. der Org., Berlin, vol. 115, pp. $825-851$. 
than would be necessary to suppress all wings; and, starting with such high concentration, intermittent light should require longer to reduce the concentration to a point at which wings are permitted to develop. In like manner, the quantity of the wing-inhibiting substance could presumably be much smaller than would barely allow all wings to develop; and from such low concentration continuous light should require longer to produce enough of the substance to stop wing production.

Only the latter of these possibilities has so far been tested by experiment. Immature aphids of the $20^{\circ}$ stock were removed at different ages from continuous light, and subjected to alternating light and darkness. By the time they were adult, and had reproduced so as to show whether all their offspring were winged, they had been in intermittent light different lengths of time. They were then returned to continuous light, and their offspring reared in successive lots. The numbers of winged and wingless individuals were used to determine the rate of return to winglessness, as outlined in an early section. Again $50 \%$ of wing suppression was the goal in the computations, and the results were as follows:

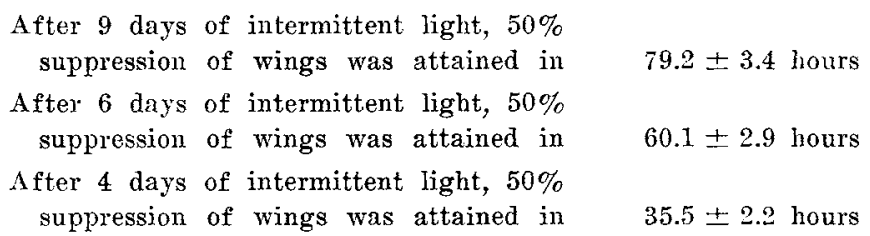

Among the last group, subjected to alternating light and darkness for 4 days, some had not in that time reached complete wing production, and such experiments were not used in computing the mean. In these exceptional ones, in the last period before the return to continuous light, a few wingless offspring were still produced. For these experiments, it was determined how long continuous light required to raise the number of wingless by $50 \%$ of the total. That is, if in the last group of offspring obtained in intermittent light there were $8 \%$ of wingless, the time required to make $58 \%$ of them wing- 
less was determined. As before, the equation of the line representing the trend toward winglessness was used in this computation, by merely substituting 58 for the term representing percentages of wingless offspring.

The mean time required for this increase from 8 to $58 \%$, from 12 to $62 \%$, from 15 to 65 and so on, was $36.4 \pm 3.3$ hours. Apparently, therefore, 4 days of intermittent light is about the minimum time which will release all wing development; and approximately a day and a half of continuous light will undo half of this effect. These statements are valid, of course, only in relation to this particular strain of aphids, and to the intensity of light used in the experiments.

The above results all tend to confirm the view that some physiological situation must be created before the light can influence wings.

In the light of the conclusion that temperature and light do not stop wing production directly, it seems entirely possible that the difference in the time required to attain that result is merely a difference in the speed of the preparatory process or processes under the influence of the two environmental agents. It is still possible, however, that the difference in the two times (71.4 hours and 24.7 hours, respectively) is made up in part of different speeds of the preparatory change, in part of different times at which wing production can be intercepted by light and by temperature. Also, it is still uncertain, as far as the preceding experiments are concerned, whether the preparatory process carried out by continuous light and that caused by high temperature are the same or different.

\section{LIGHT AND HEAT IN SUCCESSION}

Some light is thrown on the uncertainties just mentioned by experiments in which high temperature was applied at the beginning of a dark period. These experiments were started, as were the others, by subjecting aphids through most of their immature life to alternating light and darkness. After they had begun to reproduce, and so had proved the efficacy of 
intermittent light in permitting wing development, they were changed to $30^{\circ}$. But the change was made at the end of one of the 8-hour light periods instead of at the beginning, as was done in all the temperature experiments so far described. Alternating light and darkness were continued during the heat treatment. The time elapsing after the change of temperature, until a $50 \%$ reduction of winged offspring had been effected, was determined by the same method as before. Nine such experiments were performed, with the results here stated and compared with the earlier results:

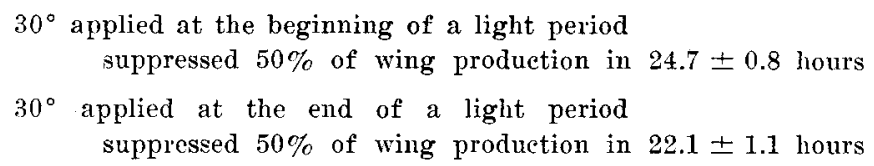

While the standard errors of these two means are large enough that the means are not proven to be significantly different, the difference is not thereby proven insignificant. It is entirely possible that they do represent actually different lengths of time required for the action of high temperature. When these experiments were performed it was not known how long continuous light alone would require to suppress half of the wing production. On the basis of earlier observations it was expected that continuous light would work much more rapidly, and the 8 hours of light prior to the application of high temperature was supposed to be a substantial fraction of the time required by continuous light alone. This latter time tumed out later to be 71.4 hours, so that the antecedent 8 hours of light in the experiments described in this section are only about one ninth of the time which continuous light alone would require. The means given above indicate a reduction of slightly more than one ninth in the time required by high temperature to stop wing production. If the reduction is not accidental, the simplest explanation of it is that the 8 hours of light had already done part of the preparatory work which the following high temperature completed. 
Similar tests were made by applying $29^{\circ}$ temperature, first at the beginning, then at the end, of an 8-hour light period. The results were as follows:

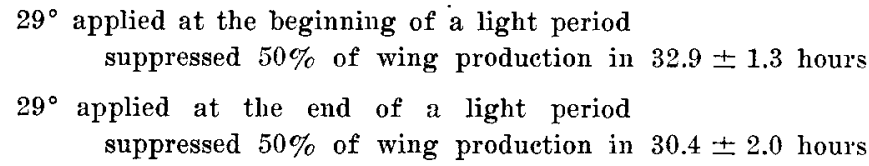

The difference is again too small to be proved significant in relation to its own standard error, and it represents a reduction of only about one thirteenth instead of one ninth; but it is in the direction which would be expected if the preceding light has done some of the same work which high temperature does.

When it was learned that in the strain of aphids being used continuous light acted more slowly than had been expected, the tests above were repeated with a much longer period of continuous light preceding the high temperature. In ten experiments, otherwise like the ones just described, the parent aphids were kept in continuous light for an average of 37.2 hours before being subjected to high temperature $\left(30^{\circ}\right)$. After such treatment only $8.7 \pm 0.9$ hours were required for the heat to complete the suppression of $50 \%$ of wing production.

The changes of duration here are not equal on a percentage basis. Continuous light was applied 37.2 hours, or $52 \%$ of the time it alone would require (71.4 hours) to suppress half of the wings. As a result, the time required by heat to finish the job of reducing wing production to half was diminished nearly $65 \%$ (from 24.7 hours to 8.7 hours). Whether the disparity of these percentages is caused by random sampling of very variable phenomena, or whether light works more rapidly in the early or middle part of its effective period than at the beginning or end, can not be judged from any of the results obtained.

From the experiments described in this section there can be little doubt that heat and light operate through the same mechanism in their control of wings. If a substance is pro- 
duced that inhibits wing development, both high temperature and continuous light bring about the accumulation of that substance.

\section{HEAT AND LIGHT IN CONJUNCTION}

Further confirmation of the conclusion just stated is afforded by the effect of heat and continuous light applied simultaneously. In the experiments first described high temperature was applied to parents which were still being subjected to alternating light and darkness. Supposedly, the effect of the heat was being opposed by the intermittent light. In another group of experiments now to be recorded heat and light were used in such a way as to accentuate each other's effect. That is, when the adult aphids were changed from $20^{\circ}$ to $30^{\circ}$, they were also changed from intermittent light to continuous light. In eleven experiments of this sort the following results were obtained:

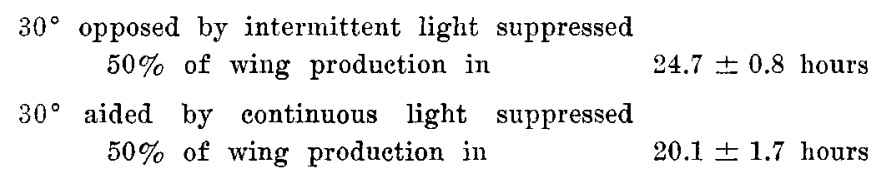

The reduction of the time required for wing suppression is not as great as might be expected. If continuous light and heat both worked at the rate which other experiments indicated, and if their effects were simply additive when they were applied simultaneously, the reduced time shown in the second line above should have been about 18.5 hours. No judgment can be formed, on any available evidence, as to whether this discrepancy is due to random sampling of variable phenomena, or to a reduction in the speed of action of heat or light, or both, when they act in unison. Either of these assumptions would seem entirely plausible.

In any case, it appears certain that heat and light are producing the same physiological change. 


\section{SUMMARY}

Continuous light, applied to aphids whose previous offspring were all winged because they were developed in intermittent light, suppressed wings in $50 \%$ of the offspring in about 3 days. High temperature $\left(30^{\circ}\right)$ applied to similarly reared aphids suppressed wings in $50 \%$ of the offspring in about 1 day. Temperatures lower than $30^{\circ}\left(29^{\circ}, 28^{\circ}, 26^{\circ}\right)$ required progressively longer time to produce the same effect. In earlier experiments, intermittent light of low intensity required longer to induce complete wing production than did light of high intensity. Aphids which have been reared in alternating light and darkness a long time (to make their offspring winged) must be treated a longer time with continuous light to suppress wings, than is necessary if the intermittent light was applied a shorter time. If aphids whose offspring are all winged, because of intermittent-light treatment, are first subjected to continuous light (of too short duration to suppress wings), the time required by subsequent heat to suppress wings is reduced. This reduction is roughly proportional to the length of time the continuous light is applied. Heat and continuous light together suppress wing production in less time than does heat with intermittent light.

These results all indicate that heat and light act through the same mechanism. If wings are suppressed by a high concentration of some substance, that substance is produced under the influence of both high temperature and continuous light. One of these agents may begin the process, the other complete it by starting at the point where the first was discontinued. The change is hastened if both agents operate at the same time. 